\title{
Appareil de contrôle en continu du tritium dans l'eau
}

\author{
S. DESCOURS et P. GUERIN (*) \\ (Manuscrit reçu le 15 mai 1980)
}

\begin{abstract}
RÉSUMÉ
L'appareil TRIDYN a été étudié pour le contrôle en continu du tritium dans les effluents avec une limite de sensibilité de l'ordre de $10^{-3} \mathrm{Ci} / \mathrm{m}^{3}$ (soit $1 / 3$ de la concentration maximale admissible en tritium dans l'eau pour la population). Il est bien adapté aux applications de la radioprotection. L'effluent est filtré, puis mesuré au passage sur un scintillateur plastique en billes, contenu dans une cellule plastique et placé entre deux photomultiplicateurs. La géométrie de la cellule et celle du scintillateur ont été étudiées pour avoir une réponse maximale. Le bruit de fond est abaissé par une protection en plomb de $5 \mathrm{~cm}$ d'épaisseur et une électronique à coïncidence du même type qu'en scintillation liquide. L'étude de la filtration et du débit sur un circuit d'eau industrielle a permis d'obtenir un temps de réponse inférieur à $30 \mathrm{~min}$ pour une concentration de $3 \cdot 10^{-3} \mathrm{Ci} / \mathrm{m}^{3}$, une tenue du scintillateur de 6 mois avec une perte de sensibilité inférieure à $10 \%$, et une autonomie de 8 jours sans intervention manuelle.
\end{abstract}

\begin{abstract}
TRIDYN has been developed for the continuous monitoring of tritium in effluents with a sensitivity limit in the range of $10^{-3} \mu \mathrm{Ci} / \mathrm{ml}$ (viz $1 / 3$ of the maximum permissible concentration of tritium in water for the public). It is well suited to health physics applications. The effluent is filtered, then measured on its passage by a ball plastic scintillator contained in a plastic cell and placed between two photomultipliers. The geometries of both the cell and the scintillator were studied in order to get the maximum response. The background was lowered by a lead shield ( $5 \mathrm{~cm}$ thick) and coincidence electronics of the same type as used in liquid scintillation counting. A study of filtration and flow rate on an industrial water circuit made it possible to obtain a response time below $30 \mathrm{~min}$ for a concentration of $3 \cdot 10^{-3} \mu \mathrm{Ci} / \mathrm{ml}$, a 6-month durability of the scintillator with a sensitivity loss below 10 p. cent, and 8-day self-operation without manual intervention.
\end{abstract}

(*) Commissariat à l'énergie atomique, Centre d'études nucléaires de Grenoble, Service de protection contre les rayonnements, 85 X, 38041 Grenoble Cedex.

RADIOPROTECTION, VOL. 16 - 0033-8451/1981/15/\$ 5.00/(C) Bordas-Dunod. 


\section{INTRODUCTION}

Le contrôle continu du tritium dans les canalisations de rejets d'effluents liquides est devenu un impératif pour tous les services de protection contre les rayonnements. Cependant, il n'existait jusqu'à ce jour sur le marché aucun appareil pouvant répondre au double problème d'un contrôle du tritium en continu dans la gamme d'activité recherchée. L'objectif recherché dans ce travail a donc été d'étudier et de faire réaliser un appareil prototype répondant à ces exigences.

Si le niveau d'activité à détecter n'est pas très faible du fait de la valeur assez élevée de la concentration maximale admissible pour la population du tritium dans l'eau $\left(3 \cdot 10^{-3} \mathrm{Ci} / \mathrm{m}^{3}\right)$, par contre, d'autres difficultés surgissent : d'une part, le mode de fonctionnement en continu a imposé une étude dynamique importante afin de minimiser le temps de réponse de l'appareil, d'augmenter son pouvoir d'autodécontamination et d'évaluer son comportement dans le temps. D'autre part, le fait que les effluents peuvent transporter d'autres contaminants radioactifs que le tritium nous a conduits à étudier la réponse de l'appareil aux principaux produits de fission. Enfin, la nature même de l'eau a une influence sur la tenue du détecteur dans le temps et nous a amenés à étudier un système de filtration.

Dans un premier temps, la réalisation d'une maquette a permis d'étudier et de définir les caractéristiques du détecteur ainsi que celles de l'électronique. Les essais ont été effectués en laboratoire, ils ont débuté par des mesures en statique puis se sont poursuivis par des mesures en dynamique. La rédaction en commun avec le Groupe de travail $\mathrm{n}^{\circ} 4$ du Comité d'instrumentation de la radioprotection (GT4/CIR) d'un cahier des caractéristiques techniques et de spécifications d'utilisation a permis la mise en fabrication du prototype.

Deux prototypes ont été réalisés au Centre d'études nucléaire de Cadarache par le Groupe d'ingéniérie d'ensembles et d'électricité du Service technique (S.T.E.C./G.I.E.E.), l'un pour le S.P.R./Grenoble, l'autre pour le réacteur EL3 au Centre d'études nucléaires de Saclay.

Ces prototypes ont permis d'effectuer des essais en vraie grandeur, à Grenoble sur des effluents liquides et à Saclay sur le circuit secondaire du réacteur EL3. Après plusieurs mois de fonctionnement réel, les conclusions tirées ont permis la mise en fabrication d'une petite série.

\section{2. ÉTUDE DE L'ENSEMBLE DE DÉTECTION}

\subsection{ESSAIS PRÉLIMINAIRES}

Toutes les difficultés dans la mesure du tritium proviennent de la faible énergie des $\beta$ émis $\left(E_{\max }=17 \mathrm{keV}, \bar{E}=6 \mathrm{keV}\right)$. Le parcours des $\beta$ de $17 \mathrm{keV}$ dans l'eau est de l'ordre du micromètre, en conséquence, seuls, les $\beta$ émis à cette distance de la surface de l'eau seront vus par le détecteur. Ce phénomène conduit 
à envisager comme seul type de mesure possible la mesure par scintillation où le scintillateur serait au contact même de l'eau à mesurer : scintillateur liquide, scintillateur plastique, anthracène...

Le niveau d'activité tritium à mesurer est de l'ordre de la concentration maximale admissible pour la population (que nous appellerons par la suite $\mathrm{CMA}_{p}$ ) soit $3 \cdot 10^{-3} \mathrm{Ci} / \mathrm{m}^{3}$. A ce niveau, il semble que le rendement d'un scintillateur plastique puisse être suffisant. Par ailleurs, le mode de fonctionnement en continu rend la mesure par scintillateur liquide mal adaptée (automatisation du prélèvement et du mélange au scintillateur). Il semble donc mieux approprié de s'orienter vers des scintillateurs solides. Ce choix étant acquis, il restait à défınir le type de scintillateur et la géométrie du détecteur, ainsi que les caractéristiques de l'électronique associée.

Les premiers essais ont pu être effectués avec des cellules à circulation du commerce (type Numelec 801 A, Numelec 808 A, et « flow-cell » de Packard), et ont conduit aux conclusions suivantes :

- Il est préférable d'utiliser une électronique à coïncidence avec deux photomultiplicateurs afin d'améliorer le rapport signal/bruit (le rendement des scintillateurs plastiques étant faible) et d'éliminer les dérives en fonction de la température. Dans ces conditions on pourra s'affranchir d'un système de refroidissement jusqu'à $22^{\circ} \mathrm{C}$.

- La sensibilité recherchée pourra alors être atteinte en optimisant les dimensions de la cellule et la géométrie du scintillateur employé sous forme de particules.

\section{2. Étude DE LA CELLULE DE DÉtection}

Les essais effectués sur les cellules du commerce ainsi que les résultats indiqués dans la littérature ont conduit à envisager le principe du scintillateur plastique sous forme de particules contenues dans une cellule en plastique qui jouerait le rôle d'adaptateur entre le scintillateur et les photomultiplicateurs $[1,2]$. Les dimensions optimales de la cellule ont été définies à partir d'une série de cellules en plexiglass réalisées dans notre atelier [3].

Pour le choix du scintillateur, les essais effectués ont conduit à éliminer successivement l'anthracène qui pose de lourds problèmes d'utilisation en dynamique (compactage, difficultés de rinçage...), les copeaux difficiles à reproduire régulièrement aux mêmes dimensions, les feuilles de scintillateur plastique utilisées par OSBORNE [1] mais difficiles à monter mécaniquement dans un boîtier, le scintillateur en bâtonnets de rendement plus faible que celui sous forme de billes.

En conclusion, le détecteur adopté comprend : une cellule en plastique cylindrique de $2,5 \mathrm{~cm}$ d'épaisseur et de diamètre égal à celui des photomultiplicateurs, rempli par $40 \mathrm{~g}$ de scintillateur plastique de type $\mathrm{NE}$ 102 A sous forme de billes sphériques de diamètre compris entre 0,6 et $0,8 \mathrm{~mm}$. 


\subsection{EsSAIS DYNAMIQUES SUR MAQUETTE}

La maquette est réalisée avec une électronique classique pour spectromètre à scintillation liquide. Il s'agit d'un modèle Packard 3002 avec des circuits de coïncidence et sommation et deux voies de mesure, l'une dans la fenêtre d'énergie $\operatorname{des} \beta$ du tritium et l'autre pour les énergies supérieures. Les photomultiplicateurs sont des RTC de type 56 DUVP/03/A. La cellule est celle étudiée précédemment.

Les essais en dynamique ont permis de vérifier :

- Que l'on pouvait atteindre un rapport signal/bruit de fond $(S / B)$ égal à 3 pour une contamination tritium de $3 \cdot 10^{-3} \mathrm{Ci} / \mathrm{m}^{3}$ (soit $1 \mathrm{CMA}_{p}$ ).

- Que le temps de réponse de la cellule pour $1 \mathrm{CMA}_{p}$ tritium est de $1 \mathrm{~min}$ et le temps de décontamination de $4 \mathrm{~min}$.

- Que l'utilisation d'une électronique avec deux voies de mesure indépendantes permet une bonne discrimination du tritium parmi d'autres contaminants.

\section{REAALISATION DU PROTOTYPE TRIDYN}

L'ensemble des résultats obtenus sur maquette a conduit à la réalisation par les services techniques de Cadarache de deux prototypes TRIDYN répondant aux caractéristiques suivantes : scintillateur sous forme de billes de NE $102 \mathrm{~A}$; cellule à circulation cylindrique $(e=25 \mathrm{~mm}, V=60 \mathrm{ml})$; photomultiplicateurs à bas bruit de fond; électronique à deux voies de type " scintillation liquide " avec une sortie calculateur, une sortie traceur, et une alarme défaut; pas de refroidissement; enceinte de plomb de $5 \mathrm{~cm}$ d'épaisseur; circuit hydraulique avec filtration et possibilité de rinçage, pour un débit de l'ordre de $20 \mathrm{l} / \mathrm{h}$.

Les performances physiques à escompter sont :

- bruit de fond inférieur ou égal à 10 impulsions/min;

- signal pour $1 \mathrm{CMA}_{p}$ tritium égal à 3 fois le bruit de fond;

- rapport des taux de comptages entre la voie " tritium " et la voie " $\mathrm{PF}$ " : égal à 10 pour le tritium, et à 0,1 pour un mélange de produits de fission.

- temps de réponse pour $1 \mathrm{CMA}_{p}$ tritium inférieur à $5 \mathrm{~min}$.

Le prototype a été conçu avec une électronique nouvelle, étudiée par le Service d'électronique et d'instrumentation (SEIn) de Cadarache, et un circuit hydraulique avec préfiltration seulement ( fig. 1 et 2 ).

\section{FONCTIONNEMENT DU TRIDYN SUR CANALISATION D'EFFLUENT}

\subsection{EsSAIS En Laboratoire}

Ils ont permis de vérifier l'étanchéité du circuit et les réglages de l'électronique. On constate que le rapport $S / B$ varie avec les paires de photomultiplicateurs ( 1,7 à Grenoble et 4 à Saclay) et que la sélection de ceux-ci 
est prépondérante. Par ailleurs, la composante des autres contaminants dans la voie tritium est égale à $1 / 8$ du taux de comptage dans la voie globale, ce qui est satisfaisant. Le temps de réponse de la cellule est un peu plus élevé que celui escompté. Mais ceci sera amélioré par une modification de la cellule sur les appareils suivants.

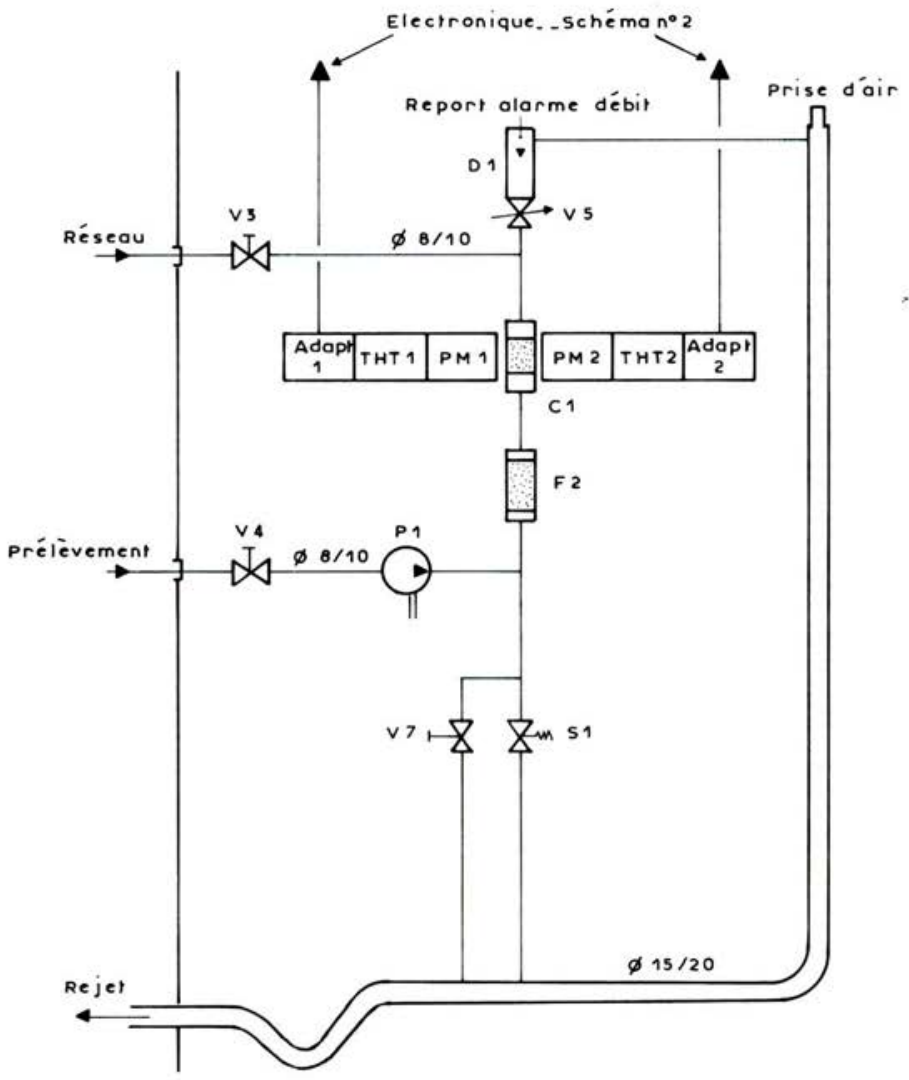

FIG. 1. - Prototype TRIDYN : circuit hydraulique.

\subsection{ESSAIS SUR ÉGOUTS « EAUX SPÉCIALES "}

\subsubsection{Description de l'installation}

L'appareil TRIDYN fonctionne en continu dans la station de contrôle de l'égout « eaux spéciales » du C.E.N. Grenoble situé à la sortie du centre ( fig. 3).

Nature de l'eau: La canalisation est alimentée à 90 p. cent du temps par l'eau du circuit secondaire du réacteur MELUSINE (et, en cas d'arrêt du 
réacteur, par de l'eau industrielle de même origine). Les caractéristiques de cette eau sont les suivantes : $\mathrm{pH} \mathrm{7,} \mathrm{charge} \mathrm{en} \mathrm{sels} \mathrm{:} 400 \mathrm{mg} / \mathrm{l}$, charge en sulfate : $55 \mathrm{mg} / \mathrm{l}$.

Point de prélèvement : A partir de l'égout, une pompe de relevage alimente un bac de décantation dans lequel sont effectués les différents contrôles d'activité. Le volume du bac est de $0,5 \mathrm{~m}^{3}$ et le niveau supérieur de l'eau est à $1 \mathrm{~m}$ du sol.

Circuit hydraulique : Le circuit de l'effluent à mesurer comprend en série : une prise sur le bac, une pompe (incorporée au châssis du TRIDYN), une unité de traitement de l'effluent, le TRIDYN proprement dit avec vanne, filtre, cellule, débitmètre,la canalisation de rejet vers l'égout.

Sortie des informations : Les signaux de sortie sont exploités par un enregistreur graphique à deux voies. Les alarmes de dépassement de seuil et l'alarme " défaut de débit " sont connectées en série sur un tableau de contrôle, mais individualisées sur l'appareil par des voyants lumineux.

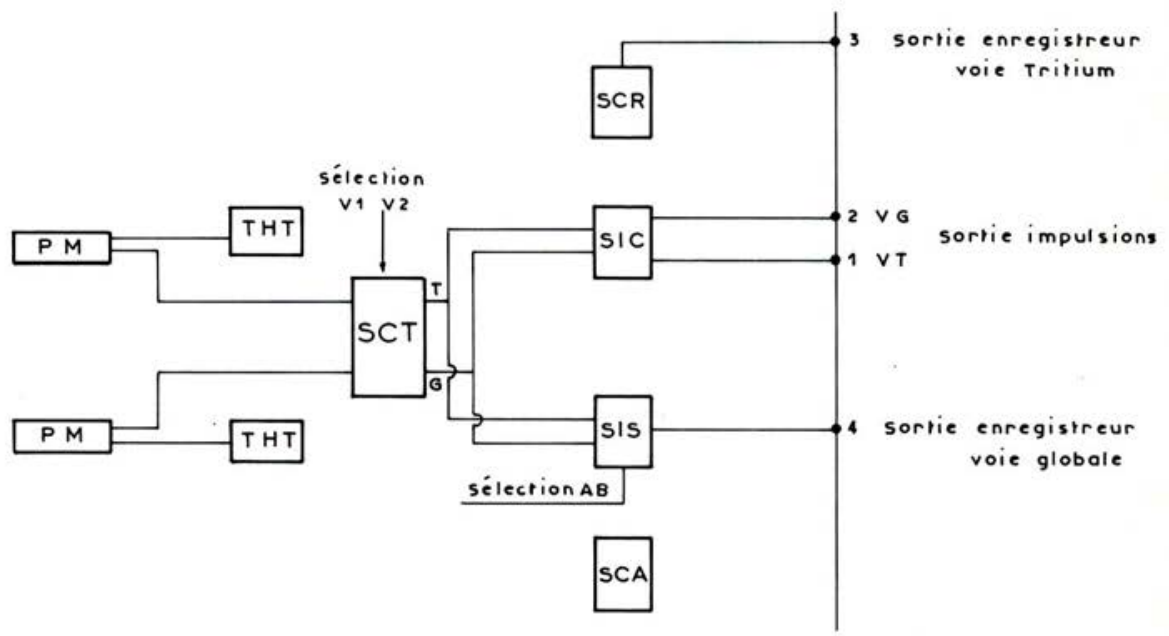

FIG. 2. - Prototype TRIDYN : schéma des modules électroniques.

\subsubsection{Essais de longue durée et traitement de l'effluent}

Il est apparu assez rapidement que le système de préfiltration prévu était insuffisant et que le scintillateur perdait ses caractéristiques principales (rendement et bruit de fond) en quelques jours. Il a donc fallu étudier un système de traitement de l'effluent plus efficace, ainsi qu'un procédé de régénération du scintillateur pollué. La régénération du scintillateur par le procédé Nuclear Enterprises (acide chlorhydrique et solution de méthanol) s'est révélé la plus efficace. Cependant, aucun procédé ne permet de restituer totalement au scintillateur ses caractéristiques initiales et ceci d'autant moins qu'il est plus 
pollué. Il est donc essentiel de bien maîtriser le traitement de l'effluent en amont du détecteur. Plusieurs méthodes ont été essayées indépendamment à Saclay, à Grenoble et à Cadarache. Le système par distillation continue et celui d'épuration par résines ont été essayés. Dans les deux cas, cela conduit à réduire considérablement le débit dans l'appareil, donc à augmenter le temps de réponse.

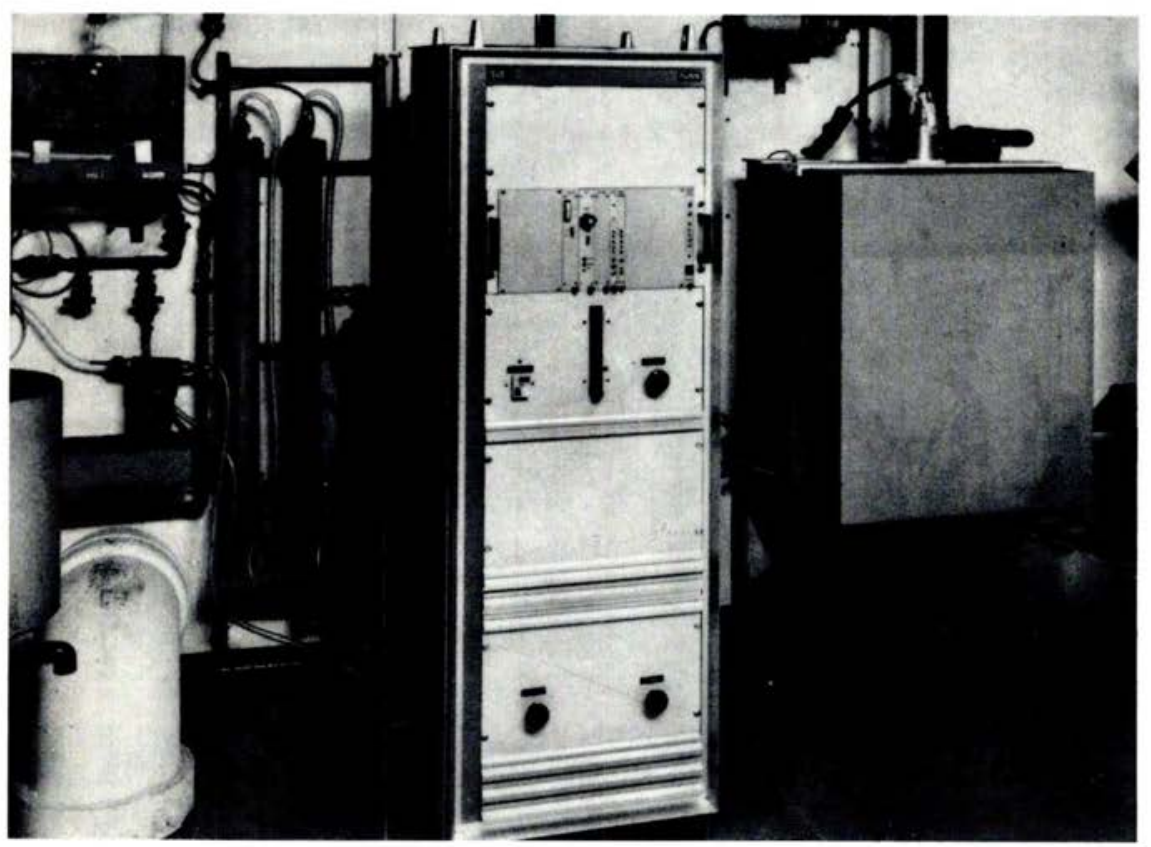

FIG. 3. - Implantation du TRIDYN sur la station d'égouts.

Il semble préférable de s'orienter vers le procédé par cartouches filtrantes mis au point par le service technique de Cadarache. L'ensemble de filtration comprend, outre le préfiltre de $0,5 \mu \mathrm{m}$ : un filtre défériseur (longueur $600 \mathrm{~mm}$ ) pour le piégeage des matières dissoutes (fer, zinc, etc.), un filtre à ions d'argent (longueur $600 \mathrm{~mm}$ ) pour le piégeage des matières organiques. Ce système autorise un fonctionnement à un débit de $20 \mathrm{l} / \mathrm{h}$ avec une perte de sensibilité du scintillateur de $10 \mathrm{p}$. cent après 2 mois de fonctionnement sans intervention. Les cartouches sont maintenant montées sur le prototype de Grenoble et fournies avec tous les nouveaux appareils.

Il faut noter que tous les types de traitement employés ont entraîné une élimination des autres contaminants radioactifs et donc amélioré la mesure du tritium. 


\section{DESCRIPTION DES NOUVEAUX APPAREILS [4]}

L'ensemble des remarques formulées par les utilisateurs des deux prototypes au cours des essais a permis au service technique de Cadarache d'apporter les modifications nécessaires aux deux appareils commandés pour le réacteur ORPHEE. Les principales améliorations ont porté sur la conception de la cellule, la protection de plomb, qui augmente de 5 à $10 \mathrm{~cm}$, le branchement de l'appareil en by-pass sur une canalisation à fort débit, le circuit de filtration, et plusieurs facilités d'utilisation comme l'accès à la cellule par écartement des coquilles de plomb, un système d'électrovannes automatiques pour fonctionner en " analyse ", " vidange " ou " test ». L'ensemble de l'appareillage est implanté dans une baie transrack IP 55-3611 avec châssis pivotant.

\section{CONCLUSION}

Cette étude a conduit à la réalisation par le service technique de Cadarache de deux prototypes puis de deux premiers appareils de série TRIDYN. L'un des prototypes est actuellement en fonctionnement sur la canalisation d'égouts " eaux spéciales » du centre d'études nucléaires de Grenoble (avant dilution dans le milieu de rejet). Les trois autres appareils, après essais sur le réacteur EL3 de Saclay, sont en cours d'installation sur le circuit secondaire du réacteur ORPHEE.

Les prototypes ont une limite de détection de l'ordre de $10^{-3} \mathrm{Ci} / \mathrm{m}^{3}$ soit un tiers de la concentration maximale admissible du tritium dans l'eau pour la population. Cette sensibilité est tout à fait suffisante vis-à-vis des exigences de la réglementation. Leur temps de réponse à une contamination tritium de $3.10^{-3} \mathrm{Ci} / \mathrm{m}^{3}$ est de $60 \mathrm{~min}$. Ce temps est dû essentiellement au procédé de traitement de l'effluent avant la mesure. L'entretien se limite à une intervention par semaine comprenant le remplacement de la cellule de détection pour effectuer le nettoyage du scintillateur.

Les deux premiers appareils de série ont des caractéristiques bien supérieures à celles des prototypes. Les modifications apportées ont permis, outre quelques facilités d'utilisation, d'abaisser la limite de détection d'environ un facteur 2 avec un temps de réponse de $30 \mathrm{~min}$. De plus, la maintenance est limitée à une seule intervention par mois.

Ce développement donné au nouveau TRIDYN en fait un appareil de contrôle en continu du tritium dans l'eau, dans la gamme d'activité de $10^{-3} \mathrm{Ci} / \mathrm{m}^{3}$ à $10^{-1} \mathrm{Ci} / \mathrm{m}^{3}$ avec une alarme pour un niveau d'activité compris dans cet intervalle. Il est donc bien adapté aux contrôles de radioprotection sur les circuits d'eaux d'égouts ou sur le circuit secondaire des réacteurs, avant rejet et dilution dans le milieu extérieur (rivières, océan, ...). 


\section{BIBLIOGRAPHIE}

[1] OSBORne R. V. Detector for tritium in water. Nud. Instr. Meth., 1970, 77, 170-172.

[2] OSBORNE R. V. Monitoring reactor effluents for tritium, problem and possibilities. Rapport AECL4054, 1971.

[3] Descours S., Guerin P. Surveillance en continu du tritium dans l'eau. Rapport CEA-R-5027, 1980.

[4] Micheletti L., Vio H. Tridyn. Note CEA/CEN/CA-STC/STEC/GIEE, n 1027-80/003, 1980.

vOL. $16-\mathrm{N}^{\circ} 1$ 